\title{
REVISED Case Report: Acute pyelonephritis and hearing loss in
}

\section{scrub typhus [version 2; peer review: 2 approved]}

\author{
Sivaranjini Venketesan11, Dheeraj Jain"1, Stalin Viswanathan (iD2, \\ Murugesan Sivagurunathan Gayathri3
}

\footnotetext{
${ }^{1}$ Department of General Medicine, Indira Gandhi Medical College \& Research Institute, Pondicherry, Puducherry, 605009, India 2Department of General Medicine, Jawaharlal Institute of Postgraduate Medical Education and Research (JIPMER)., Pondicherry, Puducherry, 605009, India

${ }^{3}$ Department of Radiology, Indira Gandhi Medical College \& Research Institute, Pondicherry, Puducherry, 605009, India
}

V2 First published: 20 Mar 2019, 8:312

https://doi.org/10.12688/f1000research.18129.1

Latest published: $05 \mathrm{Jul}$ 2019, 8:312

https://doi.org/10.12688/f1000research.18129.2

\section{Abstract}

Acute pyelonephritis is a common renal manifestation in patients with diabetes. A 52-year-old diabetic lady presented with loin pain, dysuria, and fever and urinary incontinence that had begun seven and three days prior to presentation respectively. She was treated with escalating spectra of intravenous antibiotics without improvement. Urine and blood cultures were sterile, while radiological investigations were suggestive of pyelonephritis. Mild hepatic dysfunction prompted consideration of scrub typhus and she improved with empirical doxycycline. Scrub IgM was later confirmed to be positive. In conclusion, local prevalence of systemic infections such as rickettsioses should always be considered in diabetics with fever, even if symptoms and signs otherwise suggest typical diabetes-related infections. We, therefore report a case of acute pyelonephritis caused by scrub typhus which has not been previously described in English medical literature.

\section{Keywords}

scrub typhus, acute pyelonephritis, urinary tract infection, hearing loss

\section{Open Peer Review}

Approval Status

1

2

version 2

(revision)

05 Jul 2019

version 1

20 Mar 2019

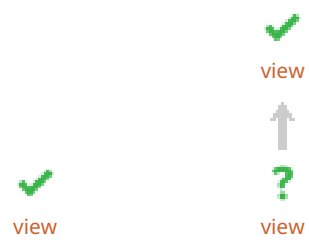

1. Dharshan Rangaswamy, Kasturba Medical

College, Manipal Academy of Higher

Education, Manipal, India

Ravindra Prabhu Attur (D), Kasturba

Medical College, Manipal Academy of Higher

Education, Manipal, India

2. Tri Wangrangsimakul ID, Mahidol

University, Bangkok, Thailand

University of Oxford, Oxford, UK

Any reports and responses or comments on the article can be found at the end of the article. 
Corresponding author: Stalin Viswanathan (stalinviswanathan@ymail.com)

Author roles: Venketesan S: Data Curation, Writing - Original Draft Preparation, Writing - Review \& Editing; Jain D: Conceptualization, Resources, Writing - Original Draft Preparation; Viswanathan S: Funding Acquisition, Supervision, Writing - Review \& Editing; Gayathri MS: Resources, Writing - Review \& Editing

Competing interests: No competing interests were disclosed.

Grant information: The author(s) declared that no grants were involved in supporting this work.

Copyright: ( 2019 Venketesan S et al. This is an open access article distributed under the terms of the Creative Commons Attribution License, which permits unrestricted use, distribution, and reproduction in any medium, provided the original work is properly cited.

How to cite this article: Venketesan S, Jain D, Viswanathan S and Gayathri MS. Case Report: Acute pyelonephritis and hearing loss in scrub typhus [version 2; peer review: 2 approved] F1000Research 2019, 8:312 https://doi.org/10.12688/f1000research.18129.2

First published: 20 Mar 2019, 8:312 https://doi.org/10.12688/f1000research.18129.1 


\section{REVISED Amendments from Version 1}

The differences are minor. History of alcohol, past treatment and hospitalizations have been mentioned. Scrub IgM kit was used and the cutoff/diagnostic values have been mentioned.

See referee reports

\section{Introduction}

Among patients with diabetes mellitus, the urinary tract is the most common site of infection ${ }^{1}$. Urinary tract infections (UTI) are either related to the upper or lower urinary tract. Acute and chronic pyelonephritis are upper UTIs ${ }^{1}$. Bacteria (Escherichia coli), viruses (Adenovirus), fungi (Mucor), and mycobacteria (Mycobacterium tuberculosis) commonly cause upper UTIs in diabetes ${ }^{1}$. Orientia tsutsugamushi (scrub typhus) has never been reported to cause pyelonephritis in English medical literature.

\section{Case report}

A 52-year-old grandmother of Indian origin, non-compliant to insulin for six months, presented to the Emergency Department of our hospital with fever and rigors, vomiting, headache, bilateral leg pain and myalgia, which had persisted for one week and urinary incontinence for the prior three days. She was unemployed, did not consume alcohol and had had no exposure to rodents or mite bites. Apart from over-the-counter antipyretics, she had neither consulted a health practitioner nor had she received antibiotics. On examination, she was conscious, oriented, toxic, febrile, drowsy, dehydrated with slurred speech, with body-mass index $20.2 \mathrm{~kg} / \mathrm{m}^{2}$, tachycardia, orthostatic hypotension, diminished hearing, with right renal angle fullness and tenderness. Initial investigations (Table 1) revealed random sugars $435 \mathrm{mg} / \mathrm{dL}$, normal renal functions, ketonuria and glycosuria without pyuria, sinus tachycardia (electrocardiogram), and normal echocardiography. There were no malarial parasites on the peripheral smear. Arterial blood gas showed respiratory alkalosis with metabolic acidosis. Intravenous ceftriaxone $2 \mathrm{~g} \mathrm{OD}$, intravenous fluids, insulin, acetaminophen 500mg three times a day, multivitamins


and folate $5 \mathrm{mg}$ ), pantoprazole $40 \mathrm{mg}$, and domperidone $10 \mathrm{mg}$ were commenced for probable acute pyelonephritis. On day 3, piperacillin/tazobactam $4.5 \mathrm{~g}$ every 8 hours and fluconazole $300 \mathrm{mg}$ once a day (OD) were substituted for ceftriaxone $2 \mathrm{~g} \mathrm{OD}$; oral amitriptyline $25 \mathrm{mg}$ was added to treat the patient's painful neuropathy.

Blood and urine cultures were sterile. Ultrasonogram showed hepatomegaly and bilateral bulky kidneys. She developed diarrhea on day 4. Hence, piperacillin was discontinued after $36 \mathrm{hrs}$ even though there appeared to be a partial defervescence; diarrhea subsided after discontinuing piperacillin. Her toxemia and prostration persisted, and she needed assistance to the toilet in view of extreme weakness, but had no focal neurological deficits. On day 5, she was initiated on meropenem $1 \mathrm{~g}$ every 8 hours and linezolid 600mg every 12 hours for persisting fever (Figure 1A). Liver function tests showed elevated transaminases; hence probable rickettsioses was suspected and empirical doxycycline $100 \mathrm{mg}$ twice a day was initiated on day 6 . The next day, Scrub Typhus Detect IgM (InBios International) done at a private laboratory returned positive with an OD of 1.732 (Cut-off: <0.500). Intravenous antibiotics were therefore discontinued and there was no recurrence of fever thereafter.

Abdominal computed tomography $(\mathrm{CT})$ on the $8^{\text {th }}$ day showed bilateral bulky kidneys with mild perinephric fat stranding

Table 1. Investigations of the patient during hospital stay and follow-up. Normal ranges for each test are provided. ESR - erythrocyte sedimentation rate.

\begin{tabular}{|c|c|c|c|c|c|}
\hline & Day 1 & Day 3 & Day 5 & Day 7 & Follow up \\
\hline Sodium (136-142 mmol/ L) & 125 & 129 & & & \\
\hline Total bilirubin) (1.1-2.4mg/dL) & & & 1.87 & & 2.25 \\
\hline Direct bilirubin (0.1-0.4mg/dL) & & & 0.3 & & 0.4 \\
\hline Total protein $(6.3-7.9 \mathrm{~g} / \mathrm{dL})$ & & & 5.2 & & 6.9 \\
\hline Albumin $(3.5-5.5 \mathrm{~g} / \mathrm{dL})$ & & & 2.8 & & 3.8 \\
\hline Aspartate aminotransferase (12-38U/L) & & & 175 & & 72 \\
\hline Alanine aminotransferase (7-40U/L) & & & 64 & & 63 \\
\hline Alkaline phosphatase (35-100U/L) & & & 299 & & 262 \\
\hline Creatinine $(0.5-0.9 \mathrm{mg} / \mathrm{dL})$ & 0.9 & & & & \\
\hline Hemoglobin (11.7-15.7g/dL) & 10.8 & 9.0 & 8.9 & 7.2 & \\
\hline Total leukocyte count (4-11 ×10\%/L) & 13.1 & 8.9 & 7.0 & 6.8 & \\
\hline Platelets $\left(150-450 \times 10^{9} / \mathrm{L}\right)$ & 202 & 311 & 362 & 368 & \\
\hline ESR (0-20 mm/hour) & 112 & 90 & 40 & & \\
\hline
\end{tabular}





Figure 1. (A) Fever spikes plotted from day 1 to day 5. (B) Computed tomography scan revealing bilateral renomegaly and mild fat stranding in the right kidney.

(Figure 1B), thus confirming the provisional diagnosis. The pancreas was normal. She also had left-sided proliferative diabetic retinopathy and bilateral sensorineural hearing loss (average of $75 \mathrm{~dB}$ and $90 \mathrm{~dB}$ in the right and left ear respectively). She then completed a 7-day course of doxycycline $100 \mathrm{mg}$ twice a day and was advised another week's therapy at home. She had had no fever thereafter. On follow up, liver function test (LFT) had improved (Table 1), as did her hearing $(47 \mathrm{~dB}$ in the right and $87 \mathrm{~dB}$ in the left) which favored our diagnosis of probable acute scrub typhus.

\section{Discussion}

Diabetes mellitus, due to hyperglycemia, ketoacidosis, vascular insufficiency, and impaired neutrophil and monocyte function, makes patients prone to UTIs ${ }^{1}$. Diagnosis of acute pyelonephritis (APN) clinically is a syndrome of fever, chills, vomiting, and flank pain associated with pyuria, and is often radiologically confirmed ${ }^{2}$. In a prospective study, only $1 / 4^{\text {th }}$ patients had a positive urine culture and only $65 \%$ had pyuria $^{2}$, echoing the findings in our patient. In total, 14 among 223 patients had 
diabetes. Even though our patient did not have pyuria, symptoms/signs in a poorly controlled diabetic led us to a diagnosis of APN and empirical treatment was instituted for the same. Renal abnormalities in scrub typhus range from simple proteinuria/hematuria to acute kidney injury and occasionally, chronic kidney disease ${ }^{3}$. Our patient had glycosuria and positive microalbuminuria $(92 \mu \mathrm{g} / \mathrm{mg})$. Mechanisms postulated for renal involvement include rickettsiae-related vasculitis, tubular interstitial proliferation, and tubular necrosis ${ }^{3}$. APN in scrub typhus has been reported only once, in Chinese medical literature in a 56-year-old Chinese lady who had urgency, flank pain and an eschar ${ }^{4}$.

Diabetes is a risk factor for scrub typhus-induced acute kidney injury. Since leukocytosis reduced with ceftriaxone without adequate fever response, we presumed poor control of bacterial/fungal infection and treated her with fluconazole and piperacillin/tazobactum. Since LFT could not be performed prior to day 4 due to technical reasons, rickettsioses were not suspected. Even though our locality is a high-prevalence area for scrub typhus, focal renal signs and symptoms led us to think otherwise ${ }^{5}$. A convalescent titer of Scrub IgM could not be done due to its unavailability and need for out-of-pocket expenses. PCR was not available. We also erred in contributing her hearing impairment to be the result of her toxemia and poor health. Pure tone audiometry was done 48 hours after doxycycline when the patient became self-ambulatory. Improvement of her hearing loss, albeit partial, two weeks after discharge suggests that scrub typhus could have also contributed to her hearing impairment ${ }^{6}$. Abdominal CT was also done after doxycycline therapy-whether findings are milder than expected is also debatable. Hypoalbuminemia and rapidly falling hemoglobin over seven days without overt blood or volume loss, could be attributed to hemoconcentration following scrub typhus-related capillary leak syndrome that was observed at initial presentation, and reverted to premorbid levels after fluid supplementation and antibiotics. In retrospect, fever, absence of pyuria, sterile urine, capillary leak syndrome, primary respiratory alkalosis, and hearing loss in a patient with high sugars and ketonuria should have made us think of an alternative etiological diagnosis. The pattern of LFT derangement did not suggest a biliary infection and pancreatic imaging was normal. Also, fever did not recur after initiating doxycycline and discontinuing other intravenous antibiotics which would favor a rickettsial infection rather than a systemic bacterial sepsis.

UTIs in diabetes are common, but scrub typhus as a probable cause of UTI/pyelonephritis has hitherto been unreported in English medical literature. Atypical organisms causing pyelonephritis should be considered in patients with multisystem involvement and in those with a UTI but without pyuria. Furthermore, local prevalence of systemic infections such as rickettsioses should always be considered in diabetics with fever, even if symptoms and signs otherwise suggest typical diabetes-related infections.

\section{Consent}

Written informed consent for publication of their clinical details and clinical images were obtained from the patient.

\section{Data availability}

All data underlying the results are available as part of the article and no additional source data are required.

\section{Grant information}

The author(s) declared that no grants were involved in supporting this work.
1. Balachandar MS, Pavkoviç $\mathrm{P}$, Metelko Ž: Kidney infections in diabetes mellitus. Diabetol Croat. 2002; 31(2): 85-103. Reference Source

2. Rollino $\mathrm{C}$, Beltrame G, Ferro $\mathrm{M}$, et al.: Acute pyelonephritis in adults: a case series of 223 patients. Nephrol Dial Transplant. 2012; 27(9): 3488-93. PubMed Abstract | Publisher Full Text

3. Hwang K, Jang HN, Lee TW, et al.: Incidence, risk factors and clinical outcomes of acute kidney injury associated with scrub typhus: a retrospective study of 510 consecutive patients in South Korea (2001-2013). BMJ Open. 2017; 7(3): e013882.

PubMed Abstract | Publisher Full Text | Free Full Text
4. Shu LH, Xu Y: [One case of scrub typhus patient with clinical manifestation of acute pyelonephritis]. Zhongguo Ji Sheng Chong Xue Yu Ji Sheng Chong Bing Za Zhi. 2013; 31(4): 329 PubMed Abstract

5. Vivekanandan M, Mani A, Priya YS, et al:: Outbreak of scrub typhus in Pondicherry. J Assoc Physicians India. 2010; 58: 24-8. PubMed Abstract

6. Premaratna R, Chandrasena TG, Dassayake AS, et al:: Acute hearing loss due to scrub typhus: a forgotten complication of a reemerging disease. Clin Infect Dis. 2006; 42(1): e6-8.

PubMed Abstract | Publisher Full Text 


\section{Open Peer Review}

\section{Current Peer Review Status:}

\section{Version 2}

Reviewer Report 05 July 2019

\section{https://doi.org/10.5256/f1000research.21739.r50808}

(C) 2019 Wangrangsimakul T. This is an open access peer review report distributed under the terms of the Creative Commons Attribution License, which permits unrestricted use, distribution, and reproduction in any medium, provided the original work is properly cited.

Tri Wangrangsimakul

${ }^{1}$ Mahidol-Oxford Tropical Medicine Research Unit, Faculty of Tropical Medicine, Mahidol University, Bangkok, Thailand

${ }^{2}$ Centre for Tropical Medicine and Global Health, Nuffield Department of Clinical Medicine, University of Oxford, Oxford, UK

The authors have revised the article and provided further useful information that improves the overall quality of the report. The lack of a convalescent sample for serological testing or other confirmatory tests remain a weakness. However, the additional details on risk factors and diagnostic testing should allow readers to draw a conclusion on the likelihood of scrub typhus infection causing pyelonephritis in this patient.

Competing Interests: No competing interests were disclosed.

Reviewer Expertise: Infectious diseases, microbiology, tropical medicine, rickettsial diseases.

I confirm that I have read this submission and believe that I have an appropriate level of expertise to confirm that it is of an acceptable scientific standard.

\section{Version 1}

Reviewer Report 28 May 2019

https://doi.org/10.5256/f1000research.19827.r48908

(C) 2019 Wangrangsimakul T. This is an open access peer review report distributed under the terms of the Creative Commons Attribution License, which permits unrestricted use, distribution, and reproduction in any medium, provided the original work is properly cited. 


\section{Tri Wangrangsimakul}

${ }^{1}$ Mahidol-Oxford Tropical Medicine Research Unit, Faculty of Tropical Medicine, Mahidol University, Bangkok, Thailand

2 Centre for Tropical Medicine and Global Health, Nuffield Department of Clinical Medicine, University of Oxford, Oxford, UK

The authors report a case of a 52 year old diabetic lady with complications, presenting with pyelonephritis. Cultures were negative and she was treated with antibiotics that were regularly escalated. She was also found to have bilateral sensorineural deafness on audiometry. Elevated liver enzymes prompted testing for scrub typhus and the IgM came back positive, leading to initiation of doxycycline and defervescence within hours.

For any case report, the diagnosis must be as robust as possible and an effort made to rule out other possible diagnoses. I agree with the other review that malaria should be ruled out. Other diagnoses to be excluded include leptospirosis, biliary infection or pancreatitis. Other background information would be useful e.g. antibiotics used pre-admission, occupation, freshwater contact, rodent contact, agricultural work, insect bites, alcohol intake and past history of scrub typhus.

The basis for the diagnosis of scrub typhus used in this report is limited. A "'Scrub IgM positive" does not inform the reader of what serological test or method was used, where the test was performed, what diagnostic cut-off was used to determine a positive result and whether this cutoff has been validated for the population which the patient belongs to. I suspect the test used was the InBios Scrub Typhus IgM ELISA, in which case the OD value and how the diagnostic cut-off was reached should be stated so the reader can form their own opinion on the strength of diagnosis.

The diagnosis of scrub typhus is not straightforward ${ }^{1}$. Antibody-detection tests (IFA, ELISAs) have inherent weaknesses, such as the presence of circulating antibodies in individuals within an endemic area where repeated exposure occurs, leading to false positive results. Acute and convalescent samples can give a clearer picture and a rise in antibody titre to $>=4$ times is usually part of the diagnostic criteria. Is a convalescent blood sample available to compare IgM serology results? Culture and PCR will be more specific and will greatly improve the certainty of diagnosis but may not be available.

Scrub typhus can cause AKI, glomerulonephritis and nephrotic syndrome so pyelonephritis is possible ${ }^{2,3}$. However, the diagnostic case for scrub typhus must be more robust than currently presented. Additionally, one could argue that the general trend was that she was improving when doxycycline was initiated on day 6 as observed on the fever chart, which would go against severe scrub typhus with a renal complication.

Two main lessons can be drawn from this report. Firstly, in regions where scrub typhus is endemic and accurate diagnostic assays are unavailable, empirical treatment with doxycycline should be considered. Secondly, antibody-detection assays for scrub typhus have inherent weaknesses and until paired antigen/IgM tests are available, acute and convalescent samples are recommended for diagnostic confirmation. In this report, the evidence for scrub typhus infection is currently limited and the conclusion that this diagnosis is established should be delayed until further diagnostic information is available. 


\section{References}

1. Paris DH, Dumler JS: State of the art of diagnosis of rickettsial diseases: the use of blood specimens for diagnosis of scrub typhus, spotted fever group rickettsiosis, and murine typhus.Curr Opin Infect Dis. 29 (5): 433-9 PubMed Abstract | Publisher Full Text

2. Rajapakse S, Weeratunga P, Sivayoganathan S, Fernando S: Clinical manifestations of scrub typhus. Transactions of The Royal Society of Tropical Medicine and Hygiene. 2017; 111 (2): 43-54 Publisher Full Text

3. Allen AC, Spitz S: A Comparative Study of the Pathology of Scrub Typhus (Tsutsugamushi Disease) and Other Rickettsial Diseases.Am J Pathol. 1945; 21 (4): 603-81 PubMed Abstract

Is the background of the case's history and progression described in sufficient detail? Yes

Are enough details provided of any physical examination and diagnostic tests, treatment given and outcomes?

Partly

Is sufficient discussion included of the importance of the findings and their relevance to future understanding of disease processes, diagnosis or treatment?

Partly

Is the case presented with sufficient detail to be useful for other practitioners? Partly

Competing Interests: No competing interests were disclosed.

Reviewer Expertise: Infectious diseases, microbiology, tropical medicine, rickettsial diseases.

I confirm that I have read this submission and believe that I have an appropriate level of expertise to confirm that it is of an acceptable scientific standard, however I have significant reservations, as outlined above.

Author Response 26 Jun 2019

Stalin Viswanathan, Jawaharlal Institute of Postgraduate Medical Education and Research (JIPMER)., Pondicherry, India

Dear Sir,

Malarial parasites were not visualized on the peripheral smear on at least 2 occasions.

Nevertheless, there was no hepatosplenomegaly, discolored urine, jaundice, hyperbilirubinemia and thrombocytopenia to suggest malaria.

The same goes for biliary infection. The lady did not have pain suggestive of pancreatitis and computed tomography of pancreas was normal. We did not perform lipase.

Leptospirosis was considered even without her exposure to rain, water, or rodents but the 
thought did not persist in the absence of renal failure and thrombocytopenia and lack of response to ceftriaxone in the first three days.

Background information regarding her employment and others have been added to the manuscript.

The diagnostic kit and the cutoff values have been mentioned in the manuscript.

Convalescence titers could not be performed due to the cost of performing it in an outside lab.

We would not generally agree that she was improving till day 5 even without doxycycline since the toxemia persisted that made us initiate meropenem and linezolid. Her symptoms did not recur on stopping these drugs and initiating doxycycline.

Culture and PCR were not available. This has been added to the text.

Competing Interests: No competing interests were disclosed.

Reviewer Report 03 April 2019

https://doi.org/10.5256/f1000research.19827.r45955

(C) 2019 Prabhu Attur R et al. This is an open access peer review report distributed under the terms of the Creative Commons Attribution License, which permits unrestricted use, distribution, and reproduction in any medium, provided the original work is properly cited.

\section{Dharshan Rangaswamy}

Department of Nephrology, Kasturba Medical College, Manipal Academy of Higher Education, Manipal, India

\section{Ravindra Prabhu Attur}

Department of Nephrology, Kasturba Medical College, Manipal Academy of Higher Education, Manipal, India

The authors describe a rare reported manifestation of Scrub Typhus namely acute pyelonephritis and hearing loss which improved with treatment. Acute pyelonephritis has been reported once as a case report from China and hearing loss in another six cases. The case is well described and convincing in that clinical features favor acute pyelonephritis. However since authors are describing a manifestation for the first time a more detailed account would have been better. For example, in view of negative cultures, treatment received prior to entry could have been listed. The authors base their conclusion on a chance sending of scrub IgM which came positive whereas learning points which serve as pointers to the diagnosis may be mentioned in discussion. The presence of liver dysfunction, anemia and fever would have merited investigation with other common tropical infections such as malaria, which can also involve the kidneys.

Unusual features in this description are absence of eschar, thrombocytopenia for scrub typhus 
and absence of pyuria. The absence of pyuria in $1 / 3^{\text {rd }}$ cases as observed in a previous prospective study, had only $6 \%$ of diabetics in their cohort and the diagnosis of acute pyelonephritis was mainly based on clinical criteria ${ }^{1}$. The sensitivity and specificity for diagnosis of acute pyelonephritis on urine investigations are: urinalysis (>10 WBCs/high power field), $58 \%-82 \%$ and $65 \%-86 \%$; positive leukocyte esterase, $74 \%-96 \%$ and $94 \%-98 \%$; nitrite, $35 \%-85 \%$ and $92 \%-100 \%$; and leukocyte esterase plus nitrite tests, $75 \%-84 \%$ and $82 \%-89 \%$ respectively $2,3,4,5$. The sensitivity and specificity for diagnosis for acute pyelonephritis based on perinephric fat stranding was $72 \%$ and $60 \%$. It is affected by age, sex and renal function and may not be a particularly useful tool for the diagnosis of acute pyelonephritis ${ }^{6}$. Bilateral enlarged kidneys, renal angle tenderness with associated vomiting are also observed in patients with infection or drug related acute interstitial nephritis. However, the absence of pyuria points against the diagnosis.

Certainly, scrub typhus can cause inflammation of organs due to endothelial infection, vasculitis and increases endothelial permeability. This report highlights the importance of keeping a high index of suspicion for this easily treatable disease especially in endemic areas. The article can be accepted as approved with caveats stated above.

\section{References}

1. Rollino C, Beltrame G, Ferro M, Quattrocchio G, et al.: Acute pyelonephritis in adults: a case series of 223 patients.Nephrol Dial Transplant. 2012; 27 (9): 3488-93 PubMed Abstract | Publisher Full Text

2. Ferry S, Andersson SO, Burman LG, Westman G: Optimized urinary microscopy for assessment of bacteriuria in primary care.J Fam Pract. 1990; 31 (2): 153-9; discussion 159 PubMed Abstract 3. Hooton TM, Stamm WE: Diagnosis and treatment of uncomplicated urinary tract infection.Infect Dis Clin North Am. 1997; 11 (3): 551-81 PubMed Abstract

4. Pollock HM: Laboratory techniques for detection of urinary tract infection and assessment of value.Am J Med. 1983; 75 (1B): 79-84 PubMed Abstract

5. Semeniuk H, Church D: Evaluation of the leukocyte esterase and nitrite urine dipstick screening tests for detection of bacteriuria in women with suspected uncomplicated urinary tract infections.J Clin Microbiol. 1999; 37 (9): 3051-2 PubMed Abstract

6. Pfaller MA, Koontz FP: Laboratory evaluation of leukocyte esterase and nitrite tests for the detection of bacteriuria.J Clin Microbiol. 1985; 21 (5): 840-2 PubMed Abstract

7. Fukami H, Takeuchi Y, Kagaya S, Ojima Y, et al.: Perirenal fat stranding is not a powerful diagnostic tool for acute pyelonephritis.Int J Gen Med. 2017; 10: 137-144 PubMed Abstract I Publisher Full Text

Is the background of the case's history and progression described in sufficient detail? Yes

Are enough details provided of any physical examination and diagnostic tests, treatment given and outcomes?

Yes

Is sufficient discussion included of the importance of the findings and their relevance to future understanding of disease processes, diagnosis or treatment?

Yes

Is the case presented with sufficient detail to be useful for other practitioners? 
Yes

Competing Interests: No competing interests were disclosed.

Reviewer Expertise: Clinical Nephrology

We confirm that we have read this submission and believe that we have an appropriate level of expertise to confirm that it is of an acceptable scientific standard.

\section{Author Response 26 Jun 2019}

Stalin Viswanathan, Jawaharlal Institute of Postgraduate Medical Education and Research (JIPMER)., Pondicherry, India

Dear Sir,

The lady did not receive any prior treatment. The details have been included.

It was not a chance sending of Scrub IgM. That facility is not available at our institution. Since LFT was done only on day 4, we could send a sample for Scrub IgM to a private lab only on day5.

Malarial parasites were not visualized on peripheral smear. Moreover, the LFT dysfunction was without hyperbilirubinemia.

All these details were not mentioned since this manuscript was submitted to another journal previously with a word count of 1000 , and such information fell prey to the editing keys.

Unusual was the absence of eschar, but it is well known in Indian populations that the incidence of eschar is varying between 4-46\% (Vivekanandan et al, 2010).

Thrombocytopenia was expected in a case of scrub typhus, and such a finding would have enabled us to pursue a diagnosis of scrub typhus earlier, even before the LFT.

Competing Interests: No competing interests were disclosed. 
The benefits of publishing with F1000Research:

- Your article is published within days, with no editorial bias

- You can publish traditional articles, null/negative results, case reports, data notes and more

- The peer review process is transparent and collaborative

- Your article is indexed in PubMed after passing peer review

- Dedicated customer support at every stage

For pre-submission enquiries, contact research@f1000.com 